\title{
Learning-based estimation of individual absorption profiles from a single room impulse response with known positions of source, sensor and surfaces
}

\author{
Stéphane Dilungana ${ }^{1}$ \\ Universite de Lorraine, CNRS, Inria, LORIA, F-54000 Nancy, France \\ Antoine Deleforge ${ }^{2}$ \\ Universite de Lorraine, CNRS, Inria, LORIA, F-54000 Nancy, France \\ Cédric Foy ${ }^{3}$ \\ Cerema, Ifsttar, UMRAE, 67200 Strasbourg, France \\ Sylvain Faisan ${ }^{4}$ \\ ICube UMR 7357, Universite de Strasbourg, CNRS, Strasbourg, France
}

\begin{abstract}
In situ estimation of the individual absorption profiles of the surfaces in a room remains a challenging problem in building acoustics. This work is aimed at studying the feasibility of this estimation in a shoebox room of known geometry, using a room impulse response (RIR) measured from a source and a sensor at known positions. This problem is tackled using supervised learning. Two neural network architectures are compared. Simulated training and validation sets of RIRs featuring various types of distortions (surface diffusion, geometrical errors and additive white Gaussian noise) are generated for the considered geometry. Two extensive empirical simulated studies are carried out to determine the influence of these distortions on the performances of learned models, and to determine which component of the RIR is most useful for absorption profiles prediction. Trained models are shown to yield errors significantly smaller than those of a naive mean estimator on every simulated datasets, including those featuring realistic perturbation levels. Our study outlines the benefit of using convolutional neural network layers, especially when geometrical errors exist. It also reveals that first order specular reflections are the most salient feature of RIRs for absorption profiles prediction under a fixed geometry.
\end{abstract}

\section{INTRODUCTION}

In building acoustics, direct in situ measurement of the absorption profiles of individual surfaces in a room remains a complex and challenging problem [1]. To tackle it in practice, the current methodology used by acousticians proceeds in two steps. First, a number of measures of the sound field are performed in the room. Second, the octave-band absorption coefficients of the different materials composing the surfaces are approximately and manually found by iteratively tuning a room acoustic simulator based on partially known room parameters, until measured values are matched. Absorption databases of known materials measured in lab conditions are also used to make sure estimated values are consistent. This approach is long, painstaking, and not always accurate.

\footnotetext{
${ }^{1}$ stephane.dilungana@inria.fr

${ }^{2}$ antoine.deleforge@inria.fr

${ }^{3}$ cedric.foy@ cerema.fr

${ }^{4}$ faisan@unistra.fr
} 
This work presents a method to estimate the absorption coefficients of the six surfaces of a shoebox room in six octave bands $(b \in[0.125, .25, .5,1,2,4] \mathrm{kHz})$ from a single room impulse response (RIR) using supervised machine learning on a large simulated RIR dataset. The method assumes that the room's dimensions and the positions of the source and receiver are fixed and known up to small geometrical errors of a few centimeters. Two neural-network architectures are compared, and several preliminary experiments on simulated datasets are carried out to assess and analyze the approach. First, its robustness to the fluctuation of three parameters affecting the sounds field is carefully studied, namely, geometrical imprecision, the presence of additive measurement noise and the presence of diffuse reverberation effects. Second, we perform a detailed analysis of which part of the RIR is most informative for the estimation, namely, the early vs. the late part for various cutoff times, plotted against the times of arrival of the direct-path and the first order specular reflections.

Learning-based room parameter estimation from a room impulse response has been the subject of two recent studies [2,3]. In our previous study [2] the surface-weighted mean absorption coefficients under arbitrary and unknown room dimensions were estimated, following a virtually-supervised learning approach that was showed to outperform classical reverberation theory formula, e.g., Sabine's law. In [3], the room dimension and absorption profiles of individual surfaces are jointly estimated using a similar learning-based approach. The profiles are given in arbitrary order, and a post assignment procedure based on the inferred geometry is introduced. However, the absorption ranges considered in the paper do not match typical building acoustic scenarios (drawn uniformly at random in $[0,1]$ ) and only three frequency bands are considered. This works takes a different approach that builds on the idea that the relative surfaces, source and receiver positions determine the times of arrival and strength of early, low-order, acoustic reflections [4,5]. Hence the frequency content of corresponding early specular reflections in the RIR retraces the acoustical properties of individual surfaces. This encouraged the proposed approach, where extraction of these cues is helped by fixing the geometry up to small fluctuations while training the model.

The remainder of this work is organized as follows. Section 2 details the RIR dataset simulation and pre-processing steps. Section 3 presents the proposed neural network architectures and training methodology. Section 4 presents the experiments and results. Finally, Section 5 concludes and outlines perspectives for future works.

\section{RIR DATASETS SIMULATION}

Supervised training of deep neural networks requires a large amount of annotated data. Since sufficiently large and diverse real RIR datasets annotated with absorption profiles are not available and impractical to measure, we follow a similar approach as [2] and generate RIR datasets annotated with corresponding absorption profiles using a room acoustic simulator.

\subsection{RIR Simulator Parameterization}

The simulator Roomsim presented in [6] is used to generate a realistic dataset of shoebox RIRs. It combines the image-source method to model specular reflections and the diffuse-rain ray-tracing algorithm to model random scattering effects. The simulated dataset consists of RIRs of duration $T=$ $500 \mathrm{~ms}$ and sampled at $f_{s}=16 \mathrm{kHz}$, corresponding to the fixed acoustic scene geometry described in Table 1. The source and receiver are simulated as omnidirectional.

Table 1: Acoustic scene geometry for datasets 1 to 6 (in meters).

\begin{tabular}{|c|c|c|}
\hline Room dimensions $[\mathrm{L}, 1, \mathrm{~h}]$ & Source location & Sensor location \\
\hline$[5.70,5.97,2.36]$ & {$[1.99,4.50,1.42]$} & {$[3.48,2.57,1.31]$} \\
\hline
\end{tabular}

The absorption and diffusion profiles of surfaces are defined by the 6 coefficients corresponding to 
Table 2: Ranges of absorption (abs) and diffusion (diff) coefficients per surface type and octave band.

\begin{tabular}{|l|c|c|c|c|c|}
\hline octave band & wall abs. & floor abs. & ceiling abs. & reflective abs. & diff. (all) \\
\hline $125 \mathrm{~Hz}$ & {$[0.01,0.50]$} & {$[0.01,0.20]$} & {$[0.01,0.70]$} & & {$[0.00,0.30]$} \\
\hline $250 \mathrm{~Hz}$ & {$[0.01,0.50]$} & {$[0.01,0.30]$} & {$[0.15,1.00]$} & & {$[0.00,0.30]$} \\
\hline $500 \mathrm{~Hz}$ & {$[0.01,0.30]$} & {$[0.05,0.50]$} & {$[0.40,1.00]$} & \multirow{3}{*}[0.01,0.12]{} & {$[0.00,0.30]$} \\
\hline $1 \mathrm{kHz}$ & {$[0.01,0.12]$} & {$[0.15,0.60]$} & {$[0.40,1.00]$} & & {$[0.20,1.00]$} \\
\hline $2 \mathrm{kHz}$ & {$[0.01,0.12]$} & {$[0.25,0.75]$} & {$[0.40,1.00]$} & & {$[0.20,1.00]$} \\
\hline $4 \mathrm{kHz}$ & {$[0.01,0.12]$} & {$[0.30,0.80]$} & {$[0.40,1.00]$} & & {$[0.20,1.00]$} \\
\hline
\end{tabular}

Table 3: Acoustic scene geometry for dataset 7 (in meters).

\begin{tabular}{|c|c|c|}
\hline Room dimensions $[\mathrm{L}, 1, \mathrm{~h}]$ & Source location & Sensor location \\
\hline$[7,5,3]$ & {$[1.7,1.4,1.6]$} & {$[3.9,2.9,1.3]$} \\
\hline
\end{tabular}

the 6 octave bands centered at $b \in .125, .25, .5,1,2,4 \mathrm{kHz}$. These bands match those available in most databases that are commonly used in building acoustic regulations. In order to obtain a diverse dataset while preserving acoustic realism, the absorption profiles are sampled using a reflectivity-biased sampling strategy similar to the one proposed in [2]. For each simulated acoustic scene, the type of each surface is drawn at random among "wall", "floor", "ceiling" and "reflective" with respective probabilities $p_{\text {wall }}=\frac{1}{3}, p_{\text {floor }}=\frac{1}{12}, p_{\text {ceiling }}=\frac{1}{12}$ and $p_{\text {refl }}=\frac{1}{2}$. The absorption coefficient of each surface in each octave band is then drawn uniformly at random depending on the surface's type inside the ranges defined in Table 2 . Those ranges are based on the absorption profiles of materials typically encountered in buildings, as found in available lab-measured databases [2]. A unique diffusion profile is drawn for all surfaces in each simulated room according to the ranges defined in Table 2. For some simulated RIRs (see Table 4), the positions of the surfaces, source and receiver are perturbed by an additive geometrical noise following the normal distribution $\mathcal{N}\left(0, \sigma^{2}\right)$ with $\sigma=0.02 \mathrm{~m}$.

\subsection{Data Preprocessing}

To suppress low frequency artifacts caused by the simulator, each simulated RIR is convolved with a high-pass filter of cutoff frequency $f_{c}=50 \mathrm{~Hz}$. For the noisy datasets, each RIR is perturbed with an additive white Gaussian noise whose standard deviation corresponds to a peak signal to noise ratio (PSNR) sampled uniformly at random in $[40,50] d B$. Finally, each RIR is scaled using max normalization, i.e., $X_{\text {normalized }}=X / \max (X)$.

\subsection{Simulated RIR Datasets}

8 annotated RIR datasets of 15,000 samples each are generated. Distortions applied on each dataset are presented in Table 4. Datasets 1 to 6 correspond to the fixed acoustic scene geometry presented in Table 1. Dataset 7 corresponds to another fixed acoustic scene geometry presented in Table 3 and is used to confront the approach to a different geometry. Dataset 8 corresponds to a dataset where the geometry of acoustic scenes is random for each observation, with room dimensions following a uniform distribution in $[3,10] \times[3,10] \times[2,5]$ and with source and sensor positions sampled uniformly in the room under the constraints of non-closeness to surfaces (1 meter) and non-mutual-closeness (1 meter). 
Table 4: Description of the simulated datasets used in this study.

\begin{tabular}{|l|l|}
\hline datasets & applied distortions \\
\hline 1 & specular reflections \\
\hline 2 & specular reflections + diffuse reflections \\
\hline 3 & specular reflections + geometrical perturbation \\
\hline 4 & specular reflections + additive white Gaussian noise (awgn) \\
\hline 5 & specular reflections + diffuse reflections + geometrical perturbation \\
\hline 6 & specular reflections + diffuse reflections + geometrical perturbation + awgn \\
\hline 7 & specular reflections + diffuse reflections + geometrical perturbation + awgn \\
\hline 8 & specular reflections + diffuse reflections + geometrical perturbation + awgn \\
\hline
\end{tabular}

\section{DEEP NEURAL NETWORK ARCHITECTURES}

\subsection{Model Designs}

Two broadly used neural network architectures are considered in this paper. The first one is a multi-layer perceptron (MLP) comporting 3 hidden layers of respectively $8000 \times 256,256 \times 128$ and $128 \times 64$ neurons. Each layer is followed by batch normalization and exponential linear units (ELUs). The second one is a convolutional neural network (CNN) composed of 4 consecutive 1Dconvolutional hidden layers of respectively 16, 32 and 64 et 128 filters of sizes 33, 17, 9,5 and a stride of 1 . Each convolutional layer is followed by a max pooling layer of width 4 , batch normalization and RELUs. The resulting output of dimension 3968 is then passed through a fully connected hidden layers of size 64 with ELUs. For each network, a final fully-connected output layer associated with a sigmoid function is used to yield the desired output vector, evaluated by a mean-squared error loss-function. Networks are optimized on the training set using a batch size of 50 and the ADAM optimizer [7] with a learning rate of 0.01 . The targeted output corresponds to the 6 coefficients of the 6 surfaces' absorption profiles resulting in a 36-dimensional vector.

\subsection{Training Methodology}

Every generated dataset is splitted into training, development and test sets corresponding respectively to $70 \%$ (10500 samples), 15\% (2250 samples) and 15\% (2250 samples) of their size. For datasets presenting noise distortion, on-the-fly noise generation on training data (referred as data augmentation) is performed during the training step to enhance the model robustness. Parameters yielding the lowest average loss on the development set over 300 epochs are used in all experiments. Early-stopping is performed when the model performances do not improve for 50 consecutive epochs, with a minimum of 100 achieved epochs.

\section{EXPERIMENTS AND RESULTS}

We first study the impact the different combinations of distortions occuring in datasets 1 to 6 have on the performances of the proposed MLP and CNN architectures. Note that for every experiment, each architecture is trained, validated and tested on training, development and test datasets with the same numbers, i.e., matched conditions but no sample overlap.

Figure 1 describes the mean absolute errors on all absorption coefficients obtained by the MLP and the CNN for datasets 1 to 6 . The performance of a naive mean estimator which always returns the mean of each absorption coefficient over the training set is also showed for reference.

First, it outlines that both the MLP and the CNN successfully learned the desired mapping, as 
they significantly outperform the mean estimator for every considered combinations of distortions, including dataset 6 which combines all of them. Moreover, the CNN is shown to significantly outperform the MLP on datasets 3,5 and 6, with sharpest improvement on dataset 3. This highlights the ability of the CNN to handle geometrical perturbations much better than the MLP. One can infer that max-pooling layers help localizing early specular reflections more robustly by selecting the highest value in convolutional windows slided across the RIRs. Finally, by comparing performances over datasets 1 and 4, one can observe that both the MLP and the CNN show good robustness to additive white Gaussian noise. Our preliminary experiments showed that on-the-fly noise generation on training data was needed to achieve this robustness.

Note that according to Figure 2, the CNN estimation errors on Dataset 6 are similar in all octave bands. Hence, we will continue presenting averaged results over all octave bands in the remainder of this section, for conciseness.

Second, we study the importance of fixing the acoustic scene's geometry for accurate absorption profile estimation. We focus on the $\mathrm{CNN}$ from now on, as it showed to be the most efficient model on the most realistic dataset 6 . Table 5 shows the mean absolute errors on all absorption coefficients obtained for datasets 6 to 8. The similar errors obtained with the CNN on datasets 6 and 7 despite their different geometry suggests that the proposed approach is independent of the specific geometry considered and can be extended to any geometry. In contrast, the CNN performances over dataset 8 (random geometry for each sample) are significantly degraded and comparable to the mean estimator. This is expected and confirms that the learned models strongly rely on fixing the known geometry of the scene in datasets. One can infer that it allows the model to learn the localization of relevant early specular reflections to estimate each absorption profile.

The last experiment studies which parts of the RIRs are most useful for the learned models. To this end, each of the 15,000 RIRs in Dataset 6 is splitted into an early part and a late part using different cutoff times. We then compare the performances of CNNs trained and tested with the early parts on the one hand and with the late parts on the other hand, for the different cutoff times. Figure 3 shows the evolution of CNN errors as a function of cutoff time for both the early and the late parts, plotted against the times of arrival of the direct path and the first order specular reflection for each of the 6 surfaces. By looking at the inflexion point of each curve, we deduce that, in accordance with intuition, first order specular reflections contain crucial information for the estimation of the absorption coefficients of their respective surface. Indeed, each surface observes a substantial improvement (resp. degradation) of its absorption profile estimation once the corresponding first order specular reflection appears (resp. disappears) in the input RIR. For all surfaces, estimation errors obtained from the early part of RIRs get close to the ones obtained from entire RIRs once both the direct path and the first order specular reflection have been observed. Nevertheless, the error keeps slightly decreasing once later parts of the RIR are included, and we note that errors obtained from late parts only, while much higher, are still significantly smaller that the naive mean estimator. This suggests that our neural models are able to retrieve information from higher-order reflections as well. However, the late diffuse parts of RIRs after the mixing time (around $50 \mathrm{~ms}$ for the considered scenes) does not seem to carry useful information for the estimation of individual absorption profiles. This again matches intuition, as sound propagation paths become mixed and indistinguishable at this stage.

Table 5: CNN mean absolute errors with $99.7 \%$ confidence intervals on absorption coefficients for datasets 6,7 and 8 .

\begin{tabular}{|c|c|c|c|c|}
\cline { 2 - 5 } \multicolumn{1}{c|}{} & Dataset 6 & Dataset 7 & Dataset 8 & mean estimator \\
\hline Mean absolute error & $0.0582 \pm 0.0007$ & $0.0682 \pm 0.0008$ & $0.1282 \pm 0.0015$ & $0.1364 \pm 0.0015$ \\
\hline
\end{tabular}




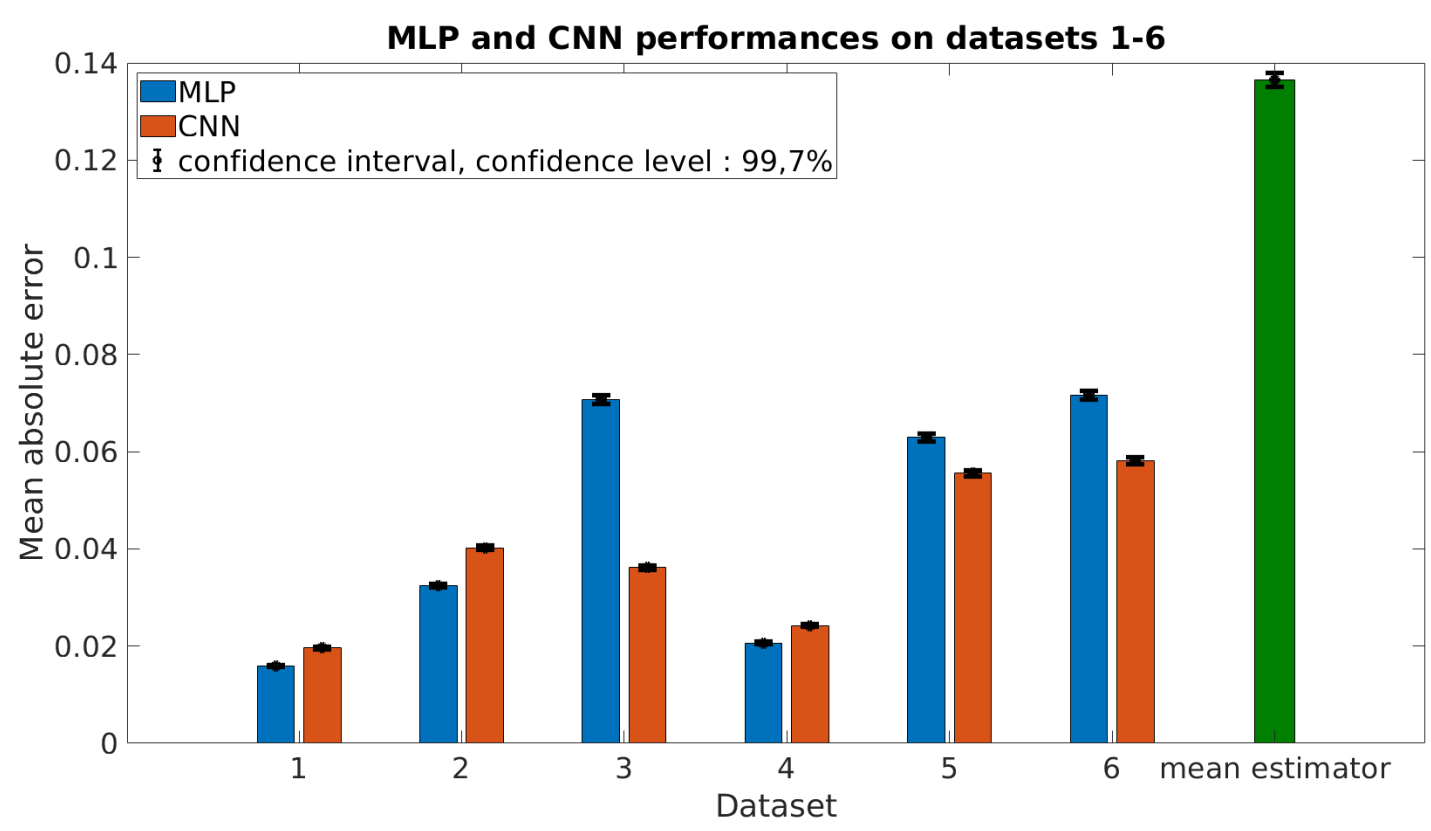

Figure 1: MLP and CNN performances on datasets 1 to 6

\section{CONCLUSION}

In this work, we tackled the inverse problem of estimating individual absorption profiles from a single RIR with known positions of source, sensor and surfaces using deep neural networks trained on simulated annotated datasets of RIRs corresponding to this geometry. Learned models are shown to significantly outperform the mean estimator whichever the set of distortions applied on data among those considered, including their combination to model realistic measured RIRs. Moreover, the CNN architecture demonstrated its efficiency compared to the MLP architecture mainly due to its noticeable robustness regarding geometrical errors. Moreover, the study reveals that the CNN performances on estimating the individual absorption profiles rely on fixing the acoustic scene geometry in the generated RIR datasets. Finally, the first order specular reflections are shown to constitute the best predictors for the individual absorption profiles of their corresponding surfaces, although some information seems to also be contained in later reflections.

Future work will include applications of the presented approach to real data. Indeed, the annotated dataset of measured RIRs presented in [5] contains multiple RIRs measured for the acoustic scene geometry described in Table 1 . New representations for the input data combining both RIR and geometrical information will be developed to make learned models efficient on datasets whose geometries vary over samples. Finally, deep generative neural networks such as Variational Autoencoders [8] which demonstrated their efficiency in solving inverse problems will be used for the considered task. 


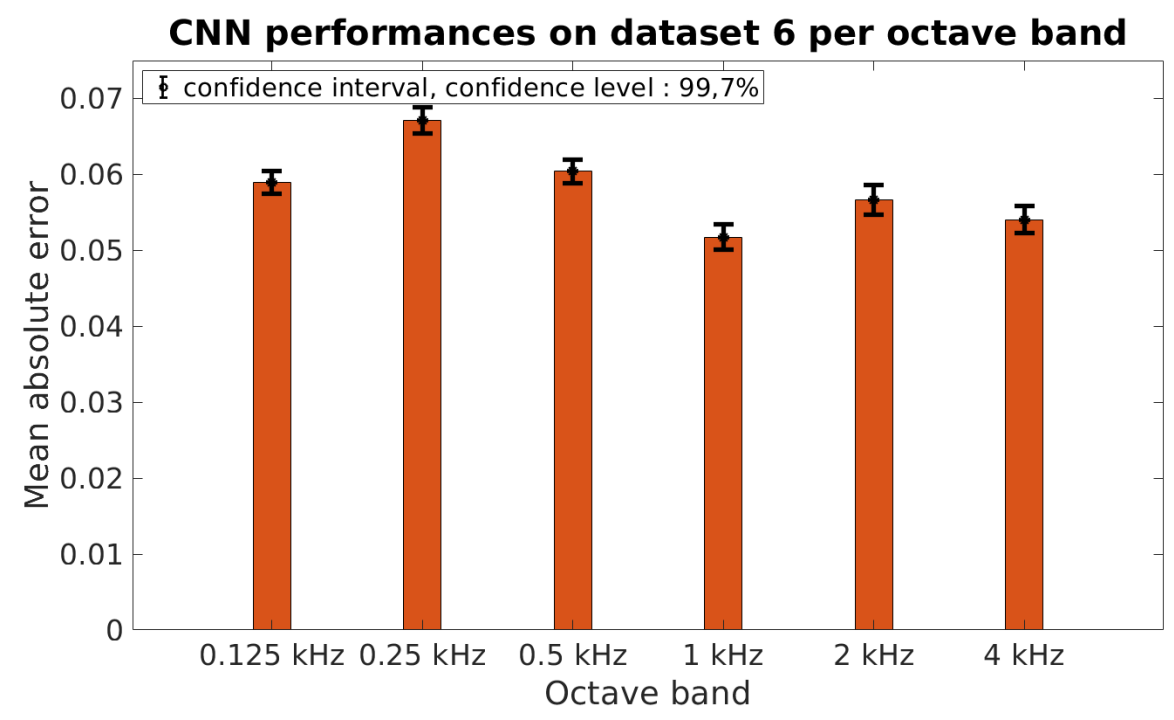

Figure 2: CNN performances on dataset 6 per octave band.

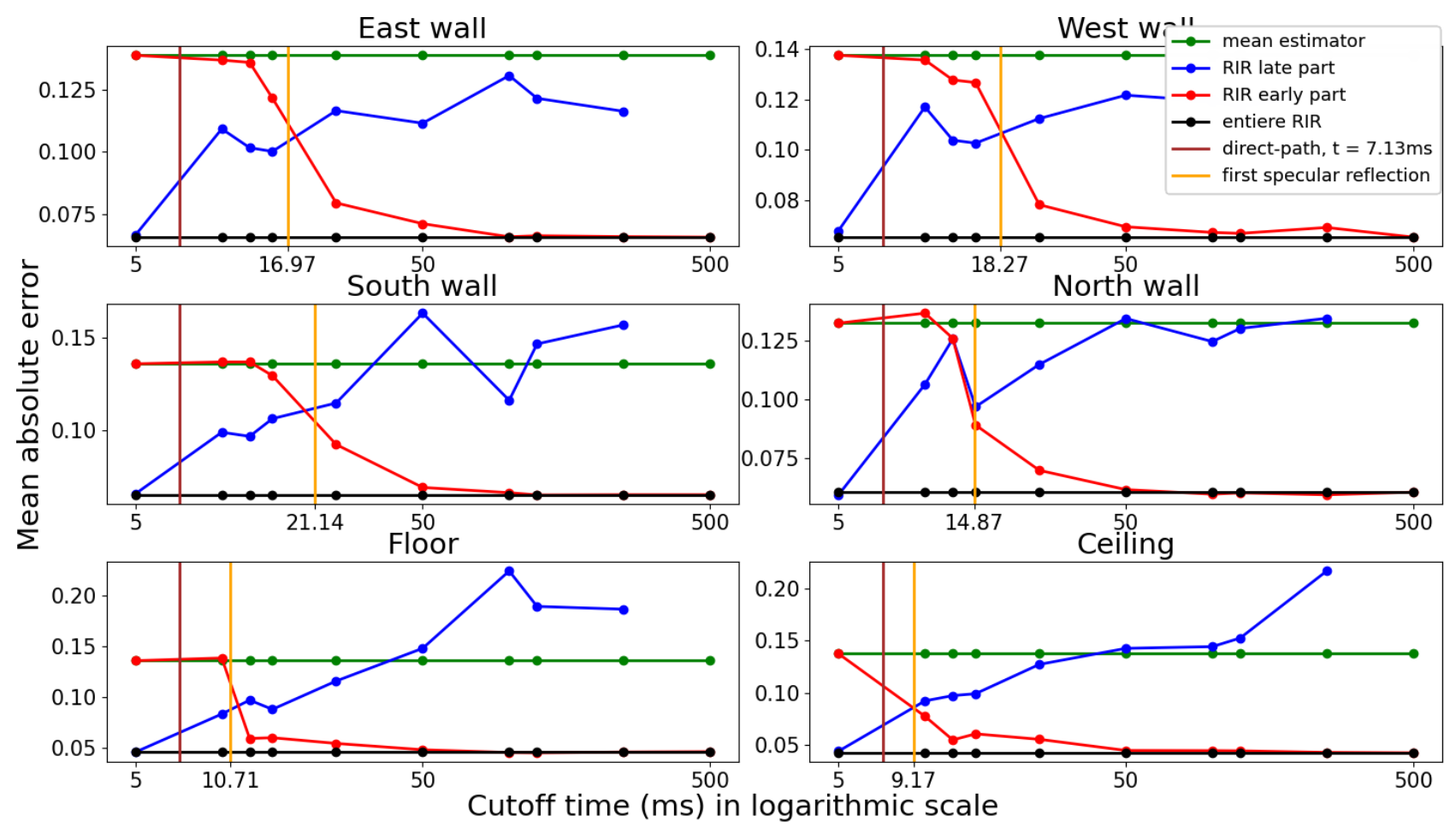

Figure 3: Mean absolute estimation errors obtained by the CNN on the absorption profiles of the 6 surfaces of the rooms in dataset 6 . Errors using only the early part or only the late part of the RIRs with different cutoff times are compared. The times of arrivals of the direct path and of the first specular reflection calculated with the Image Source Method applied on Table 1 geometry are indicated for each surface. 


\section{REFERENCES}

[1] Eric Brandão, Arcanjo Lenzi, and Stephan Paul. A review of the in situ impedance and sound absorption measurement techniques. Acta Acustica united with Acustica, 101(3):443-463, 2015.

[2] Corto Bastien, Antoine Deleforge, and Cédric Foy. Mean absorption coefficient estimation from impulse responses: Deep learning vs. sabine. In Forum Acusticum 2020, 2020.

[3] Wangyang Yu and W Bastiaan Kleijn. Room acoustical parameter estimation from room impulse responses using deep neural networks. IEEE/ACM Transactions on Audio, Speech, and Language Processing, 29:436-447, 2020.

[4] Ivan Dokmanić, Reza Parhizkar, Andreas Walther, Yue M Lu, and Martin Vetterli. Acoustic echoes reveal room shape. Proceedings of the National Academy of Sciences, 110(30):1218612191, 2013.

[5] Diego Di Carlo, Pinchas Tandeitnik, Cédric Foy, Antoine Deleforge, Nancy Bertin, and Sharon Gannot. dechorate: a calibrated room impulse response database for echo-aware signal processing. arXiv preprint arXiv:2104.13168, 2021.

[6] Steven M Schimmel, Martin F Muller, and Norbert Dillier. A fast and accurate "shoebox" room acoustics simulator. In 2009 IEEE International Conference on Acoustics, Speech and Signal Processing, pages 241-244. IEEE, 2009.

[7] Diederik P Kingma and Jimmy Ba. Adam: A method for stochastic optimization. arXiv preprint arXiv:1412.6980, 2014.

[8] Diederik P Kingma and Max Welling. Auto-encoding variational bayes. arXiv preprint arXiv:1312.6114, 2013. 